CP, 2013 , N², pp. 31-40. ISSN 2014-6752. Girona (Catalunya). BAS PEÑA, Encarnación y BARRÓN, Margarita: INCLUSIÓN EDUCATIVA DE COLECTIVOS DESFAVORECIDOS. EDUCACIÓN Y GÉNERO. UNA MIRADA DESDE ARGENTINA.

Recibido: 03/01/2013 - Aceptado: 21/01/2013

\title{
INCLUSIÓN EDUCATIVA DE COLECTIVOS DESFAVORECIDOS. EDUCACIÓN Y GÉNERO. UNA MIRADA DESDE ARGENTINA.
}

\section{Educational inclusion of disadvantatged groups. Education and gender. A glance from Argentina.}

\section{Autoras: BAS PEÑA, Encarnación y BARRÓN, Margarita}

Directora del Departamento de Teoría e Historia de la Educación.

Universidad de Murcia

Directora de la carrera de posgrado "Especialización en Adolescencia con mención en Educación y Psicología de Desarrollo".

Universidad Nacional de Córdoba (Argentina)

ebas@um.es /margarita@barron.com.ar

\section{Resumen}

Este trabajo forma parte del Proyecto de Cooperación interuniversitaria "Inclusión educativa de colectivos desfavorecidos. Educación y género", obtenido en la "I Convocatoria de proyectos de Cooperación al Desarrollo UMU-CAJAMAR”. Recogemos el trabajo realizado por la Universidad Nacional de Córdoba (Facultad de Filosofía y Humanidades. Argentina) y la Universidad de Murcia (España).

Analizamos el cumplimiento de las leyes argentinas sobre educación y género en los centros educativos CENMA, las percepciones del profesorado y del alumnado respecto a la igualdad y la violencia de género, y las medidas más utilizadas para promover la igualdad y prevenir la violencia de género.

Los resultados muestran un escaso conocimiento sobre las leyes argentinas referidas a equidad de género y prevención de la violencia de género, cuestiones no tratadas habitualmente en estos centros, el profesorado precisa materiales y formación para integrarlas en el currículo de forma continua y sistemática.

\section{Palabras clave}

\begin{abstract}
This work is part of the inter-university Cooperation Project "Inclusión educativa de colectivos desfavorecidos. Educación y género", obtained in the "I Convocatoria de proyectos de Cooperación al Desarrollo UMU-CAJAMAR". We collected the work of the National University of Córdoba (Faculty of Philosofy and Humanities. Argentina) and the Universty of Murcia (Spain).

We analyzed compliance with Argentine's laws about education and gender in CENMA schools, the perceptions of teachers and students on equality and gender violence, and the measures used to promote equality and prevent gender violence.

The results show a lack of knowledge on Argentine laws regarding gender equality and prevention of gender violence, issues that have not been previously treated in these centers hence, the teachers need materials and training to integrate it continuously and systematically within the curriculum.
\end{abstract}

\section{Key words}

education, gender, violence, adolescence. 


\section{Introducción}

Esta investigación se ha llevado a cabo en Córdoba (Argentina) como parte del proyecto de Cooperación Interuniversitaria "Inclusión educativa de colectivos desfavorecidos. Educación y género", obtenido en la "I Convocatoria de proyectos de Cooperación al Desarrollo UMU-CAJAMAR", (Resolución Rectoral 664/2010, U. de Murcia) en el que han participado las siguientes universidades de Argentina: Universidad Católica de Córdoba. Facultad de Educación. Universidad Nacional de Córdoba. Facultad de Filosofía y Humanidades. Universidad Nacional de Río Cuarto; y la Universidad de Murcia (España). Presentamos el trabajo realizado por la Universidad Nacional de Córdoba (Facultad de Filosofía y Humanidades) y la Universidad de Murcia (Facultad de Educación).

Marchesi (2012) anuncia que en la sociedad y la educación iberoamericanas la pobreza y la desigualdad están presentes de forma acusada en muchos países, lo que afecta a la educación, a la igualdad en su acceso, y a la falta de opciones laborales para un amplio colectivo de la juventud.

Las Metas Educativas 2021: la educación que queremos para la generación de los Bicentenarios pretende lograr que la educación, como un derecho fundamental y un componente básico del desarrollo humano, contribuya a conseguir la inclusión de los colectivos desfavorecidos, y la igualdad entre hombres y mujeres, tal y como señalan los Informes internacionales y, concretamente, los relativos a América Latina y el Caribe. En este sentido, el informe Miradas sobre la educación en Iberoamérica 2012, señala el género entre los factores que continúan provocando la exclusión o la discriminación en el acceso a la educación o el éxito en la finalización de los estudios emprendidos (pp. 68).

También, el último Informe anual del Observatorio de igualdad de género de América Latina y el Caribe: El salto de la autonomía. De los márgenes al centro (2011: 78), indica que "uno de los desafíos que enfrentan los mecanismos para el adelanto de la mujer es el de poder seguir avanzando en la institucionalización de la transversalización de género, en el sentido de que el enfoque de género permee profundamente las culturas institucionales más allá de las personas o equipos responsables en cada repartición estatal”. En materia de legislación es necesario superar la precariedad en las garantías de acceso a derechos presentes en las constituciones, las leyes y los instrumentos internacionales (pp. 8).

En la investigación centramos nuestra atención en la población adolescente de 14 a 25 años que asiste al centro educativo CENMA 215, en la zona norte de la ciudad de Córdoba. Los CENMA son escuelas nocturnas de enseñanza media para adultos abiertas ahora a adolescentes, a partir de los 14 años, que habían abandonado el sistema educativo formal. Es un colectivo expuesto a altos riesgos, de acuerdo con los resultados de anteriores investigaciones (Barrón, Bas, Crabay y Schiavoni, 2010). Revisamos la legislación argentina referida a educación y género, su implementación en el CENMA 215; las medidas más utilizadas para promover la igualdad y prevenir la violencia de género; así como las percepciones del profesorado y del alumnado respecto a estas cuestiones. Para ello hemos utilizado una metodología multimétodo, cuantitativa y cualitativa, de acuerdo con los objetivos de la investigación.

Los resultados muestran que aunque Argentina cuenta con un marco legal adecuado sobre género y educación, sin embargo no era conocido por el profesorado ni el alumnado de estos centros, antes de ésta investigación, por lo que la formación del profesorado es un factor fundamental para implementarlas, mediante medidas educativas integradas en el curriculum que promuevan la reflexión y toma de conciencia sobre la desigualdad, provocada por el género.

Finalmente expresamos nuestro agradecimiento a todas las personas que han hecho posible este trabajo, sin ellas nunca hubiera visto la luz. 


\section{Objetivos}

\author{
1. Analizar las leyes argentinas referidas a educación y \\ género.
}

2. Identificar experiencias educativas relacionadas con educación y género.
3. Conocer la posición del profesorado y del alumnado respecto a la igualdad y la violencia de género.

\section{Metodología}

Se trata de una investigación aplicada, destinada a explorar aspectos demográficos y posturas frente a la equidad de género y la violencia de género entre la juventud que asiste al CENMA 215. Cuenta con una sede central (CENMA madre) a la cual se fueron incorporando, con el transcurso del tiempo, varios centros anexos ( 5 en total) y una extensión áulica. Ofrece una educación de nivel primario y secundario, tanto presencial como semipresencial. Su singular estructura y la multiplicidad de ofertas educativas le otorga a la institución una organización compleja, y una organización social multifacética constituida por un entrecruzamiento de realidades, poblaciones, con un funcionamiento en muchos aspectos diferentes y en otros comunes. La Institución trabaja con diferentes programas sociales tales como el Plan Jefas y Jefes de Hogar, la terminalidad educativa para madres adolescentes, el Programa de Inclusión Social, entre otros; y, por otro, que la mayoría del profesorado trabaja en diferentes sedes.

Hemos manejado una metodología multimétodo, cuantitativa y cualitativa, de acuerdo con los objetivos de la investigación y las fases establecidas. Para la recogida de información utilizamos la revisión documental, encuestas, grupos de discusión y entrevistas. El universo de estudio estuvo formado por directivos educativos (3), profesorado (6), y alumnado de entre 14 y 25 años (350 adolescentes) que asistieron, durante 2010 y 2011, al CENMA 215, en la zona norte de la ciudad de Córdoba. Es un colectivo expuesto a altos riesgos, que habiendo desertado previamente de la escuela media se reinsertan en ella a través de esta modalidad. Las unidades de análisis fueron los riesgos psicosociales de estas personas y las cuestiones de género.

La técnica utilizada en la recolección de datos cuantitativa consistió en la autoadministración del cuestio- nario sobre conductas de riesgo asociadas a morbimortalidad en la adolescencia, ya utilizado por nuestro equipo de investigación (Barrón, 2005, 2006). A posteriori se realizaron entrevistas con alumnado, docentes y directivos para investigar el grado de conocimiento relacionado con la existencia de las leyes sobre educación y género. Por último, se realizaron dos grupos focales con el alumnado, en los que se trabajó sobre proyectos de vida, violencia, consumo de alcohol y otras drogas, sexualidad y género, igualdad de oportunidades para varones y mujeres y prevención de la violencia de género, y conocer las situaciones problemáticas que cada grupo priorizaba. También se realizaron seis relatos de vida a sujetos puntuales.

El procesamiento de datos se realizó mediante el programa Epi-Info con una confiabilidad estadística del $95 \%$. Se efectuó un análisis cuanti y cualitativo de los datos. Se realizaron entrevistas, grupos focales y talleres con jóvenes objeto de estudio. Se discriminaron los aspectos sociodemográficos a los fines de caracterizar los grupos de jóvenes que asistían a estos establecimientos.

\subsection{Fases seguidas}

FASE 1: a-Analizar las leyes argentinas referidas a educación y género, ya indicadas anteriormente. bIdentificación experiencias educativas relacionadas con educación y género.

Esta fase incluyó la recopilación de las leyes sobre educación y género y las gestiones administrativas ante el Ministerio de Educación de la Provincia de Córdoba para acceder al CENMA 215, sus docentes y alumnado para conocer cómo están aplicando la legislación. Para ello, se realizó una encuesta a responsa- 
bles educativos, alumnado, padres y madres, entrevistas y grupos focales.

FASE 2. Identificación de las medidas más utilizadas para promover la igualdad y prevenir la violencia de género.

Se llevó a cabo una búsqueda bibliográfica y documental (principales bases de datos, y artículos de las revistas de impacto internacionales de ISI), orientada a:

a) materiales sobre coeducación e igualdad entre hombres y mujeres o sobre resolución no violenta de los conflictos, utilizados en los centros educativos de Córdoba.

b) programas puntuales sobre coeducación y fomento de la igualdad que se aplican en los centros de Córdo$\mathrm{ba}, \mathrm{y}$ formación de los profesionales que los desarrollan.

c) Programas de formación específica del profesorado que los lleva a cabo.
FASE 3. Trabajo Campo. Valoración de las percepciones del profesorado y del estudiantado respecto a la igualdad y la violencia de género.

Se ejecutaron las siguientes acciones: 3 entrevistas a directores o directoras, 6 a integrantes del profesorado. Relatos de vida a 6 alumnos/as. Grupos focales (2).

Este Trabajo de Campo permitió elaborar un perfil de los conocimientos y expectativas que, sobre la temática, poseían docentes y alumnado, así como planificar la implementación de acciones educativas específicas.

FASE 4. Difusión y transferencia de los resultados.

Las reuniones de los equipos se realizaron en la forma prevista en lo que se refiere a viajes. Sin embargo es muy importante destacar que mucho trabajo se realizó también en el intercambio constante entre los equipos a través de internet en sus diversas formas (email, Messenger, videoconferencias, dropbox). Asimismo, se generaron espacios para presentar públicamente los resultados, fase en la que nos encontramos en estas fechas.

\section{Estado de la cuestión o antecedentes}

Los antecedentes del presente estudio se remontan al curso 2006-07, fecha en la que se sientan las bases mediante acuerdos interinstitucionales para el desarrollo de proyectos de gestión educativa, investigación y docencia entre estas universidades. Se firmaron convenios ILA (Intercambio con Latinoamérica) con la U. Nacional y la U. Católica de Córdoba (Argentina) y, desde entonces, se promueve el intercambio interuniversitario. En 2008, en la convocatoria de ayudas para la realización del programa de cooperación interuniversitaria e investigación científica entre España e Iberoamérica (AECI, Resol. 11620, junio de 2008) obtuvimos la Acción Complementaria Seminario para la elaboración del plan de estudios del postgrado en Educación Social, en el marco del Espacio Iberoamericano de Educación Superior (PCI: C/017709/08).

Esta investigación refleja la consolidación de la cooperación interuniversitaria, experiencia que trasladaremos en un futuro al modelo de cooperación triangular. En ella recogemos los datos más relevantes para contribuir al desarrollo del conocimiento científico que permita: a) conocer en qué medida y de qué manera se están implementando las leyes argentinas referidas a educación y género, en estos centros; b) identificar las medidas más utilizadas para promover la igualdad y prevenir la violencia de género; c) analizar las percepciones del profesorado y del estudiantado respecto a la igualdad y la violencia de género.

La pertinencia, actualidad y relevancia del trabajo que presentamos se puede constatar en las recomendaciones tanto de las conferencias sobre la mujer realizadas (ONU), como por diferentes Informes. Señalamos, además de los indicados anteriormente, el Informe sobre Desarrollo Humano para MERCASUR 2009-2010. Innovar para incluir: jóvenes y desarrollo humano; el Informe de CEPAL (2007) sobre Cohesión social: inclusión y sentido de pertenencia en América Latina y el Caribe; la Undécima Conferencia Regional sobre la Mujer de América Latina y el Caribe (2010); el Informe de actividades del Observatorio de Igualdad de Género de América Latina y el Caribe, 2008-2010; y, el 
último Informe anual 2011, del Observatorio de igualdad de género de América Latina y el Caribe (OIG): El salto de la autonomía. De los márgenes al centro que señala que "en materia de legislación, si bien existen avances heterogéneos en algunas áreas, como en la lucha contra la violencia de género, es necesario superar la precariedad en las garantías de acceso a derechos presentes en las constituciones, las leyes y los instrumentos internacionales" (pp. 8), y afirma que "la misma educación es un factor de empoderamiento y de superación de la pobreza" (pp. 42).

En Argentina, a partir de 2002 y como corolario de la firma de tratados internacionales, surgen leyes sociales de derecho tales como: -Ley 23.179, de Ratificación de la Convención sobre la Eliminación de todas las Formas de Discriminación contra la Mujer. -Ley 23.849, de Ratificación de la Convención de los Derechos del Niño. -Ley 25.212. Ratificación del Pacto Federal del Trabajo. -Ley 25673. Ley de Programa Nacional de Salud Sexual y Procreación Responsable.-Ley 26.061- Ley de Protección Integral de los Derechos de las Niñas, Niños y Adolescentes. -Ley 26.150- Programa de Educación Sexual Integral. -Ley 26.206- Ley Nacional de Educación.- Ley 26.390- Prohibición del Trabajo Infantil y Protección del Trabajo Adolescente.- Lineamientos curriculares para la educación sexual integral (Programa Nacional De Educación Sexual Integral. Ley Nacional No 26.150)- Ministerio de Educación- Consejo Federal de Educación- Mayo de 2008.

En Argentina existe actualmente el marco legislativo necesario para la incorporación de la perspectiva de género. Otra cuestión es su conocimiento para implementarlas en sus prácticas educativas profesionales y conseguir las Metas de 2021.

\subsection{Marco teórico o referentes conceptuales}

Krauskopf (2005) señala que las sociedades latinoamericanas están fraccionadas debido a la inequidad, la exclusión y la pobreza. Quienes no acceden a la educación, se les facilita una identidad negativa. Están expuestos a la explotación laboral, a emigrar más rápidamente, a llevar una vida ociosa... El embarazo temprano, muchas veces es considerado el principal proyecto de vida, la forma de alcanzar reconocimiento social. La situación de la mujer es especialmente crítica en estas condiciones. La producción de soledades, separa y aísla a cada quien de sus potencias, poniendo una vez más de manifiesto el anillado indisoluble de un modo de producción con los diversos modos de objetivaciónsubjetivación que en él se despliegan. Castells y Subirats (2007) definen el mundo de las mujeres como "una cultura propia hecha de observación subordinada y comportamiento estratégico a partir de una información más variopinta que la de los hombres y de los atributos en que tenían ventaja comparativa, desde la seducción hasta el socorro del frágil ego masculino".

La construcción de las identidades no responde a determinismos biológicos sino culturales, es una construcción sociocultural elaborada a partir de discursos ideológicos y normativos que articulan, vertebran, codifican y perfilan nuestras percepciones, experiencias, conductas y sexualidad. Las personas, en tanto que sujetos sociales, forjan su identidad en sociedad a partir de imágenes y representaciones culturales que son impuestas desde la infancia y entre cuyos márgenes deben configurar su existencia. En palabras de Sartre, "soy algo que no he elegido ser" (Sartre, 1989: 380). La estructura patriarcal se ha apropiado de las diferencias anatómicas entre los sexos para configurarlas como una deficiencia sobre la que legitimar unas relaciones sociales asimétricas entre hombres y mujeres. Por consiguiente, la jerarquización y subordinación es el resultado de unas categorías de percepción construidas en torno a un sistema de oposiciones binarias, que remiten a las diferencias anatómicas entre ambos sexos, y configura la forma de ver el mundo, nuestro pensamiento y el imaginario colectivo, proyectándose en el sistema político, legislación y políticas públicas.

Es preciso que reflexionemos sobre nuestra forma de pensar y la manera en que los demás nos piensan; deberíamos deconstruir lo que Bourdieu denominó "habitus", es decir, el sistema de categorías de percepción, pensamiento y acción (Bourdieu, 2005) para obtener la autonomía. Si pretendemos dar algunas respuestas satisfactorias a las nuevas realidades sociales conviene que nos cuestionemos constantemente la validez de muchos conocimientos, de los procedimientos utilizados, de las aportaciones de 
las investigaciones, de las propias experiencias y actuaciones.., porque la educación es construida y recreada, día a día según las ideas, los intereses, las creencias, los valores, las percepciones y los sentimientos de quienes se involucran en ella.

Marchesi, Tedesco y Coll (2012:7) destacan la importancia de la educación y de la igualdad para superar los desafíos a los que se enfrenta "la sociedad y la educación iberoamericanas: asegurar el bienestar de su ciudadanía, el desarrollo económico y la cohesión social en un mundo que vive profundas y aceleradas transformaciones...”. Como señala el Informe sobre desarrollo humano para MERCASUR 2009-2010. Innovar para incluir: jóvenes y desarrollo humano: "En materia de políticas públicas, el enfoque de género ha hecho explícita la necesidad de enfrentar las situaciones de discriminación y desigualdad, para lo cual se requieren medidas concretas en la familia y el hogar, en el mundo laboral y en los derechos políticos, en salud, salud sexual y reproductiva, y educación. La adopción de este enfoque conecta directamente con el reconocimiento de los derechos de las mujeres, la preocupación por la ampliación de la ciudadanía y la responsabilidad cívica" (pp. 256). Los datos de las investigaciones e Informes internacionales ponen de relieve que son las mujeres y las niñas las que sufren mayor pobreza y desigualdad (Bas y Amador, 2010).

En Occidente a pesar de la igualdad legal obtenida, la igualdad real continúa siendo una asignatura pendiente, y las mujeres suelen ser "ciudadanas de segunda”. Como afirma Mayor Zaragoza (2001:149): "incluso en los casos en que la sociedad la reconoce como sujeto, la mujer sigue siendo, la mayoría de las veces, una "liberta". La ruptura de la servidum- bre aún no ha llevado, ni siquiera en los países más avanzados, al pleno disfrute de los derechos, a la libertad y la igualdad efectivas". Por tanto, defendemos una educación que promueva el pensamiento, la autonomía, la aceptación y el respeto a las diferencias, la imaginación y la creatividad para buscar nuevas formulas que ayuden a resolver los problemas que surgen en la convivencia personal y grupal, que lleve a fundamentar las posiciones sin dogmatismos, a comprender los conflictos desde una perspectiva positiva, que promueva el cambio para el crecimiento como seres humanos y miembros de una sociedad basada en los derechos humanos. No una educación que adoctrine, que anule la capacidad de analizar, de contrastar, de pensar y de tomar decisiones de acuerdo con unos principios.

Se necesita lo que Morin (2011:151) llama "conocimiento pertinente" que no es más pertinente porque contenga más información o esté muy organizado matemáticamente, sino porque se ubica en su contexto con el que está relacionado (relacionando lo abstracto con lo concreto), mostrando las diferentes caras de una misma realidad. El conocimiento parcelado produce ignorancias globales, porque conduce a importantes desconocimientos. Es preciso superar la fragmentación del conocimiento, reformar el pensamiento para que sea pueda "relacionar los conocimientos entre sí, de relacionar las partes con el todo y el todo con las partes, un pensamiento que pueda concebir la relación de lo global con lo local, de lo local con lo global" (pp. 141), ya que el conocimiento es sólo conocimiento en tanto es organización, relación y contextualización de la información” (Morin, 2008:16).

\section{Resultados, Análisis y Discusión}

La participación del alumnado fue masiva, rica y comprometida. Entre los directivos hubo también un alto grado de participación e interés. En el profesorado podemos distinguir dos grupos, uno se comprometió ampliamente con el trabajo, y el otro no estuvo interesado en participar. Señalamos algunos de los resultados obtenidos de:

1. Las encuestas efectuadas:La distribución de la población joven (14 a 25) del CENMA 2015, por edad y sexo, es la siguiente: Entre 14 y 17 años, el 22,2\% son varones y el 27,7\% mujeres; de 18 a 21 años prácticamente se mantiene el porcentaje $(22,7 \%)$ de varones, mientras que se reduce más de un $10 \%$ el porcentaje de mujeres $(16,6 \%)$; $y$, finalmente entre $22-25$, los varones representan el 5,5\%, mientras que no hay ninguna mujer. Observamos, en los datos totales, un ligero incremento de varones, aunque lo más llamativo es la mayor presencia de mujeres en el tramo de menor edad. Situación que 
podemos relacionar con los embarazos prematuros, pues existe un 55,5\% de embarazos en mujeres muy jóvenes. Se declaran personas activas sexualmente el 94,4\%, sólo usan preservativo el $50 \%$, y hay un $27,7 \%$ de abortos.

El 77,7\% conviven en la casa con su padre y con su madre. El 22,2\% sólo con la madre, mientras que no hay ningún caso que conviva con el padre.

Respecto a las situaciones de violencia observamos diferentes situaciones y la necesidad de intervenir educativamente para trabajar en la resolución pacífica de los conflictos personales e interpersonales, que tienen lugar en este colectivo. El 11,1\% lleva navaja, el 55,5\% ha participado en peleas, el $11,1 \%$ ha estado en prisión por peleas, $y$ el $33,3 \%$ ha visto o participado en peleas en el centro educativo.

El consumo de drogas es otra cuestión relevante para trabajar con esta población por las consecuencias que tienen en su vida y en sus relaciones con su entorno. El 66,6\% consumen tabaco y bebidas alcohólicas, el 22,2\% psicofármacos, y el 16,6\% cocaína.

La formación especifica del profesorado es una necesidad palpable para que integren en sus prácticas educativas profesionales acciones orientadas a prácticas saludables respecto a la sexualidad, el consumo de drogas, la gestión pacífica de situaciones problemáticas.

2. Las entrevistas, grupos focales y relatos de vida, realizadas para conocer las percepciones de los directivos educativos de estos centros, profesorado y estudiantado respecto a la igualdad y la violencia de género, nos aportan los siguientes datos:

a. Con los directivos y profesorado

Las entrevistas realizadas al director del CENMA 215, a los coordinadores de cada sede y docentes de las diversas asignaturas que accedieron a entrevistarse con el equipo de investigación, reflejan que:

- El conocimiento sobre las leyes argentinas citadas es limitado, estando más presente la ley de educación y la de educación sexual que las referidas a equidad de género y prevención de la violencia de género.
- Se están implementando las propuestas de la Ley Nacional de Educación. En relación a las temáticas específicas, sólo se están implementando acciones de educación sexual en un tercio de las escuelas y no está incluida en lo curricular en los CENMA.

- En cuanto a equidad de género y violencia de género no son temas abordados habitualmente, salvo que surja puntualmente en la institución una situación insoslayable. En esos casos se generan iniciativas en cada institución y en algunas circunstancias - como por ejemplo la trata de personas que surge como tema prioritario en los medios de comunicación- pero no están aún articuladas en los temas, ni hay prácticas curriculares comunes.

- Cuando se profundiza el diálogo con el profesorado entrevistado sobre las Medidas más utilizadas para promover la igualdad y prevenir la violencia de género, se observa ausencia de preparación sobre coeducación e igualdad entre hombres y mujeres o sobre resolución no violenta de los conflictos, tampoco están formando los profesionales que los desarrollen a través de Programas de formación específica del profesorado.

- Las escuelas entrevistadas realizan anualmente talleres para tratar temas como abuso de alcohol y drogas, salud sexual y reproductiva, pero no se centran en acciones para reforzar los Factores de Protección que favorezcan la adquisición de habilidades comunicativas, el desarrollo de su capacidad de escucha, su expresión verbal y no verbal, capacidad de resolución de problemas, percibirse competente para la actividad escolar, desarrollar la autoestima, creatividad, sentido del humor, autonomía responsable, tolerancia a las frustraciones, capacidad de posponer la gratificación, manejo de la rabia -enojo y de las emociones en general, para desarrollar la empatía. Incluso las acciones que se realizan están supeditadas a la presencia de invitados, que se ofrecen a ejecutarlas, pero no cuentan con espacios curriculares propios y, por tanto, están supeditados a la disposición de tiempo que puedan ceder las materias curriculares. No tienen continuidad en el tiempo, lo que les resta efectividad.

Algunas instituciones trabajan, desde algunos espacios curriculares, en el análisis de propagandas, video clips, películas, canciones discutiendo actitudes, valores y conductas frecuentes entre los ado- 
lescentes y jóvenes que asisten a esas escuelas, con la intención de proporcionar experiencias de aprendizaje interesante y aplicable a su realidad cotidiana, pero no hay un trabajo organizado, sistematizado y transferible.

\section{b. Con el alumnado del CENMA}

En las entrevistas y grupos focales con el alumnado comenzamos indagando sus principales preocupaciones, que resultaron ser el acceso a planes y subsidios, la posibilidad de acceder a trabajo estable al finalizar los estudios en la escuela media, la violencia en general, el fácil acceso a las drogas, el frecuente uso de marihuana y cocaína entre el alumnado, el acceso a planes de salud sexual y reproductiva.

- La violencia de género no fue mencionada espontáneamente como una de sus prioridades para ser tratada. Tampoco se señalaron cuestiones relacionadas con desigualdad de las mujeres.

- Al plantear la educación como un factor para superar la desigualdad de la mujer, su contribución productiva y participación en la toma de decisiones, se manifestaron confusos, planteando que no era un tema en el que hubieran pensado mayormente, y que la escuela no había tratado anteriormente este tema, de forma clara. Se señaló que existían planes sociales especialmente orientados a las mujeres.

- Si bien reconocían como frecuente la violencia de género, plantearon no haber participado nunca en un debate, al no ser un tema instalado en el grupo. Aceptaron que podía ser de interés ver material audiovisual, como punto de partida para abordar y reflexionar en grupo sobre esta cuestión.

- Las mujeres presentes participaron activamente de los comentarios, aunque se mantuvieron en una posición muy general, sin entrar en situaciones concretas.

- Se habló libremente del abuso de consumo de alcohol y drogas, tanto por varones como mujeres, de las características de consumo en cuanto al tipo, cantidad y frecuencia. Puestos a reflexionar sobre el tema señalaron que al carecer se seguridades para el futuro, ver escasas oportunidades laborales, dificultades para compartir algo estable con una pareja, el acceso a sustancias se convierte en un paliativo.

- En cuanto a la violencia insisten en que está presente en sus vidas de forma constante, incluso con detenciones por la policía por participar de peleas y otros hechos de violencia, que se dan tanto en los varones como en las mujeres.

- Aceptan que la violencia de género existe y señalan que muchas veces se desencadena en relación a negativas de la mujer a quedar embarazada, hecho que en ciertos sectores se considera menoscaba la masculinidad de la pareja.

- Los relatos de vida realizados por alumnas, dan cuenta de la frecuencia de embarazos tempranos, que determinaron su abandono de la escuela media, de las dificultades de pareja, de trabajo, de inclusión social, de dependencia de subsidios del gobierno y de los planes alimentarios, así como de su baja autoestima y escasas expectativas de futuro.

Finalmente, respecto a la legislación sobre equidad de género y prevención de la violencia de género, tanto el profesorado como el alumnado, manifestaron no haber "escuchado hablar de esas leyes", "no creen que sean aplicables a su medio", van a ser difíciles de hacer cumplir", "va a pasar mucho tiempo hasta que estén vigentes porque van contra lo que ocurre en la realidad día a día".

\section{Conclusiones}

Los datos ponen de relieve que estos centros realizan anualmente talleres para tratar cuestiones como abuso de alcohol y drogas, salud sexual y reproductiva, pero no están integradas en el curriculum, sino realizadas por personas invitadas que se ofrecen a realizarlas. Por tanto, no tienen continuidad en el tiempo, y es dudosa su efectividad.

Se evidencia un escaso conocimiento sobre las leyes argentinas referidas a equidad de género y prevención de la violencia de género. La equidad de género y la violencia de género no están incluidos en el curriculum, no son temas abordados habitualmente, salvo que surja puntualmente por una situación problemática. En esos casos, pueden surgir iniciativas, según cada institución, pero no ha coordinación entre ellas, ni se realizan prácticas comunes.

El profesorado no conoce ni utiliza Medidas para promover la igualdad y prevenir la violencia de géne- 
ro. Se evidencia ausencia de formación y de materiales sobre coeducación e igualdad o relacionados con la resolución no violenta de los conflictos.

El alumnado no mencionó espontáneamente, en las entrevistas y grupos focales, la violencia de género como una de sus prioridades. Aceptan que la violencia de género existe y señalan que muchas veces se desencadena en relación a negativas de la mujer a quedar embarazada, porque en ciertos sectores se considera que afecta a la masculinidad de la pareja.

Consideramos muy positiva la ejecución de este proyecto, ya que desnudó reticencias sociales a la equidad de género y a la prevención de la violencia, especialmente contra la mujer.

Cabe señalar entre las repercusiones de esta investigación el haber generado conocimiento de la legislación sobre equidad de género y prevención de la violencia de género entre el amplio número de personas que han participado en esta investigación, y en el grupo de personas de 26-70 años que asisten al CENMA
215, y con los que tienen lazos familiares. Además, ha hecho visible a la mujer en la comunidad y entre los actores de la misma escuela, así como la desigualdad por género y las situaciones de violencia que sufre la mujer, marcando un antes y un después.

Con esta investigación se ha generado la necesidad de formación en género y derechos humanos que se proporcionará tanto en la Licenciatura de Ciencias de la Educación, como en los cursos de postgrado y en la carrera de Especialización en Adolescencia, en la que se realizará una página web, con la intención de crear una red.

Se implementarán cursos en el nivel medio, asesoramiento a autoridades institucionales universitarias y otras. Se tiene previsto realizar jornadas y cursos de sensibilización y capacitación. Se trabajará con los liderazgos de jóvenes para que sean ellos quienes desarrollen también la prevención. También se prevé transferir esta experiencia a otras instituciones educativas.

\section{Referencias}

- Barrón, M. (Comp) (2005): Inequidad sociocultural, riesgo y resiliencia. Córdoba (Argentina), Brujas.

- Barrón, M. (Comp) (2006): Violencia. Córdoba (Argentina), Brujas.

- Barrón, M., Bas, E., Crabay, M. y Schiavoni, C. (2010): "Adolescentes, violencia y familia en la ciudad de Córdoba (Argentina)". Pedagogía Social. Revista Interuniversitaria, $\mathrm{n}^{\circ} .17$, pp. 83-95.

- Bas, E. y Amador, L. (2010): "Mujer y exclusión social”, en Intervención social y género. Madrid, Narcea.

- Bourdieu, P. (2005): La dominación masculina, Barcelona, Anagrama.

- Castells, M. y Subirats, M. (2007): Mujeres y Hombres. Madrid, Alianza.

- Krauskopf, D. (2005): “Comprensión de la juventud. El ocaso del concepto de moratoria psicosocial". JOVENES, Revista de Estudios sobre Juventud. Año $8, n^{\circ}$. 21. México, DF, julio-diciembre, pp.26-39.
- Marchesi, A., Tedesco, J.C. y Coll, C. (Coord.) (2012): Calidad, equidad y reformas en la enseñanza. OEI. Madrid, Santillana. Colección Reformas educativas. Metas Educativas 2021: la educación que queremos para la generación de los Bicentenarios

- Mayor Zaragoza, F. (2001): Un mundo nuevo. Barcelona, Círculo de lectores.

- Morin, E. (2008): La cabeza bien puesta. Repensar la reforma. Reformar el pensamiento. Buenos Aires, Nueva visión.

- - (2011): La vía. Para el futuro de la humanidad. Barcelona, Paidós.

- Sartre, J. P. (1989): El ser y la nada, Barcelona, Losada.

Recursos electrónicos:

- Marchesi, A. (2012): "Presentación", en Miradas sobre la educación en Iberoamérica 2012, OEI, en $<$ http://www.oei.es/miradas2012.pdf>

[Consultado: 02/11/2012]. 
- Mercasur 2009-2010. Innovar para incluir: jóvenes y desarrollo humano. PNUD, en <http://www.undp.org.ar/nov195.html> [Consultado: 11/10/2012].

- Comisión Económica para América Latina (CEPAL) (2007): Cohesión social: inclusión y sentido de pertenencia en América Latina y el Caribe, en $<$ http://www.oei.es/quipu/cohesion_socialAL_ CEPAL.pdf $>$

[Consultado: 10/10/2012].

- - Informe de la Undécima Conferencia Regional sobre la Mujer de América Latina y el Caribe, celebrada en Brasilia, del 13 al 15 de julio de 2010, en <http: / / www.eclac.org/cgibin/getprod.asp?xml $=/$ mujer $/$ noticias $/$ paginas $/ 5 / 38885 / \mathrm{P} 38885 . \mathrm{xml}$ $\& \mathrm{xsl}=/ \mathrm{mujer} / \mathrm{tpl} / \mathrm{p} 18 \mathrm{f} . \mathrm{xsl} \&$ base $=/ \mathrm{mujer} / \mathrm{tpl} /$ top $->$

[Consultado: 07/10/2012].
- - Informe de actividades del Observatorio de Igualdad de Género de América Latina y el Caribe, 2008-2010, en

<http://www.eclac.org/mujer/publicaciones/xm 1/2/39992/InformeActividades.pdf $>$

[Consultado: 14/10/2012].

- - Informe anual 2011 del Observatorio de igualdad de género de América Latina y el Caribe (OIG): El salto de la autonomía De los márgenes al centro, en <http: / /segib.org/colaboraciones/files/2011/08 /2011-242-OIG-Informe_anual_WEB.pdf> [Consultado: 05/12/2012].

- OEI. (2012). Metas Educativas 2021. La educación que queremos para la generación de los bicentenarios. Informe final, en <http://www.oei.es/metas2021.pdf > [Consultado: 10/12/2012].

\section{Forma de Citación}

BAS PEÑA, Encarnación y BARRÓN, Margarita: Inclusión educativa de colectivos desfavorecidos. Educación y género. Una mirada desde Argentina. Revista Communication Papers, No 2, páginas 31 a 40. Departamento de Filología y Comunicación de la Universidad de Girona. Recuperado el _ de de 2 de: http://www.communicationpapers.es 